however, there are not enough studies in the pediatric population to show the influence of treatments on the immune system., and therefore, that justify this clinical practice.

Objectives: To analyze the levels of T, B and NK lymphocyte subpopulations in patients diagnosed with JIA treated with FAMES and biological therapy.

Methods: A descriptive and cross-sectional study in which 39 patients from the Pediatric Rheumatology Unit of the Reina Sofía University Hospital were recruited, diagnosed with JIA according to the ILAR 2001 criteria. The patients were divided into four groups: 8 controls in clinical remission without treatment, 17 in treatment with DMARD in monotherapy, 7 in biological treatment in monotherapy and 7 in treatment with DMARD-biological. Patients with systemic JIA were excluded because they had a pathogenic mechanism different from the rest of the JIA categories. By flow cytometry, the levels of CD3, CD4, CD8 and CD19 cells were measured for acquired immunity and from NK for innate, the CD4/ CD8 index was also calculated.

Results: The mean age of the 39 patients was $10 \pm 5.7$ years, 29 were girls $(74.3 \%), 4$ patients had arthritis related to enthesitis, 16 patients had oligoarticular involvement ANA +, 6 subjects polyarticular involvement FR- and 13 they were psoriatic arthritis. Although no statistically significant differences were found when contracting cellular levels among the 4 groups evaluated, it was observed that the group treated with DMARD monotherapy had the highest percentage of children with alterations in cellular levels CD3, CD4, CD8 and CD19 (41.17\% of the patients of the group); the group treated in monotherapy with biological treatment $(28 \%)$ presented alteration in the levels of CD8 and CD19 and the group treated in combination of DMARD and biological (14.28\%) in CD19. On the other hand, the NK cells and the CD4/CD8 index were not altered in any of the groups. Only 6 cases of serious infections were registered in patients in combination therapy (DMARD-biologic) who had received corticosteroids by clinical activity. There were no statistical differences between patients who had received corticosteroids and those who did not.

Conclusion: Patients in treatment with DMARD monotherapy had a tendency to decrease cellular levels. On the other hand, alterations in innate immunity or CD4/CD8 index were not observed.

Disclosure of Interests: None declared

DOI: 10.1136/annrheumdis-2019-eular.3753

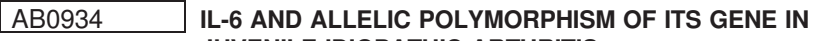 JUVENILE IDIOPATHIC ARTHRITIS}

Agar Artsymovych, Olena Oshlianska. Shupyk National Medical Academy of Postgraduate Education, Paediatrics №1, Kyiv, Ukraine

Background: The development and maintenance of inflammation in juvenile idiopathic arthritis (JIA) is mediated by cytokine imbalance; interleukin 6(IL6) plays a leading role among pro-inflammatory cytokines. Its pathological synthesis has a negative impact on all organs and system. It is not excluded that its effector ability depends on genetic structures of IL6 gene. It has not been studied whether the allelic polymorphism of the IL6-174CG gene affects the effectiveness of targeted biological therapy. Objectives: to assess the IL6 dynamics level in serum of patients with ineffective JIA-treatment.

Methods: The level of IL6 in the serum of JIA patients 1-18y.o. was determined using ECLIA method, debut of the disease (54) and treatment failure (36: 6 oJIA, 14 pJIA, 10 enthJIA, 6 sJIA). In patients with JIA, allelic polymorphism of the IL6-174CG gene was studied by PCR-method using allele-specific primers. A correlation analysis of clinical and laboratory parameters was made.

Results: Among patients with ineffective treatment of JIA, the duration of the disease was $39.5 \pm 35.8$ months, $62.9 \%$ were girls. 27 patients received GC (<1 mg/kg), 30-MTX(10-15mg/m2), 3-leflunomide, 1-AZA, 10TZ, 16-ADA/ETA, 1-TFC, 5 were switched from antiTNF for antill6. The level of IL6 in the serum of JIA patients with treatment ineffectiveness was higher than at the beginning of disease (sJIA $52.40 \pm 73.84 \mathrm{vs} 24.4 \pm 4.7$ ( $<<0.05$ ); pJIA 36.19 $\pm 58.6 v s 2.3 \pm 0.25$ (p <0.05); OJIA $8.69 \pm 5.27 v s 1 \pm 0.1$; enthJIA $90.55 \pm 63.33 \mathrm{vs} .4 \pm 0.9 \mathrm{pg} / \mathrm{ml})$. There was no increase in IL6-level in patients with an unfavorable course of the RFpos-pJIA $(8.4 \pm 7.35 \mathrm{pg} / \mathrm{ml})$ and with uveitis $(5.59 \pm 4.94 \mathrm{pg} / \mathrm{ml})$ (norm.1.5-7pg/ml). In $57.1 \%$ of cases of RFneg-pJIA IL6 was elevated, in 3 children it was the highest (79.77$218.7 \mathrm{pg} / \mathrm{ml})$, they had anemia and osteoporosis. A high $(51-484.6 \mathrm{pg} / \mathrm{ml})$ level was observed in 4 patients with enthJIA, in 1-inadequate therapy was performed, in 3 patients there was a fever, a lag in physical development, the highest CRP (up to $300 \mathrm{mg} / \mathrm{ml}$ ). The correlation between the level of IL6 in the blood of children with JIA and the presence of lymphadenopathy in the debut of the disease $(r=0.53)$, the child's age at the debut of the disease $(r=0.63)$, examination $(r=0.74)$, and the patient's weight $(r=0.87)$, the duration from the onset of the disease to the initiation of biological therapy $(r=0.44)$, the number of exacerbations in the first years of the disease $(r=0.66-0.69)$, the formation of contractures and the limitation of movement in the joints $(r=0.75)$, radiological progression in the 1st year from the debut $(r=0.54)$, ESR $(r=0.48)$, CRP $(r=0.40)$, doctor's estimate of disease activity $(r=0.79)$, ALT $(r=0.69)$, AST $(r=0.99)$, $\mathrm{LDH}(r=0.73)$ was found. There was no correlation with the number of affected joints $(r=0.28)$, heart rate $(r=0.48)$, metabolic abnormalities on the ECG ( $r=0.39)$. In children with an adverse course of JIA, IL6 correlated backward with TNF $(r=-1)$, which was not observed in children at the onset of the disease $(r=0.19)$.

Analysis of the results of genetic examination showed that among children with treatment failure $50 \%$ had GC-allele of the IL-6-174GC gene, $27,3 \%$ had GG allele, and $23,7 \%$ had CC allele.

Conclusion: Treatment of JIA leads to changes in cytokine profile. The IL6-174CG gene is associated with a high level of IL6 in the blood. The longer the duration of the disease and the time before the start of treatment with biological DMARDs, the greater the likelihood of an increase of IL-6-level in serum. In cases of even short-term enzymemia in JIA subclinical activity with IL-6 can be suggested. In case of presence of some signs (hyperthermia, osteoporosis, anemia) in patients with antiTNF, it is advisable to determine the level of IL-6 in blood serum.

Disclosure of Interests: None declared

DOI: 10.1136/annrheumdis-2019-eular.2414

\section{AB0935 ANTI-TIF-1 G-ANTIBODIES IN JUVENILE DERMATOMYOSITIS ARE ASSOCIATED WITH VARIOUS CLINICAL PHENOTYPES}

Brigitte Bader-Meunier ${ }^{1}$, Cyril Gitiaux ${ }^{2}$, Jean-Luc Charuel ${ }^{3}$, Nicole Fabien ${ }^{4}$, Isabelle Melki ${ }^{5}$, Pierre Quartier ${ }^{6}$, Alexandre Belot ${ }^{7}$, Nathalie Streichenberger ${ }^{4}$ Christine Bodemer 6 , Audrey Aussy ${ }^{8}$, Olivier Boyer ${ }^{8} .{ }^{1}$ Hôpital Necker, Pediatrics, Paris, France; ${ }^{2}$ Hopital Necker, Pediatrics, Paris, France; ${ }^{3}$ Hôpital La Pitié, Immunology, Paris, France; ${ }^{4} \mathrm{HCL}$, Lyon, France; ${ }^{5}$ Hôpital R.Debré, Paris, France; ${ }^{6}$ Hopital Necker, Paris, France; ${ }^{7}$ HFME, Lyon, France; ${ }^{8}$ Rouen University Hospital, rouen, France

Background: Juvenile dermatomyositis (JDM) is a rare heterogeneous autoimmune disease. The identification of myositis specific antibodies (MSAs) has allowed the characterization of subgroups of JDM patients who each have specific phenotypes. Antibody $(\mathrm{Ab})$ against transcriptional intermediary factor-1- $\gamma$ (TIF-1- $\gamma$ or $\mathrm{p} 155 / 140)$ is the most common MSA in JDM ${ }^{1}$. In the American and English JDM cohorts, anti-TIF-1- $\gamma$ associated JDM is classically associated with a larger proportion of caucasians, mild or moderate severity with typical cutaneous manifestations (Gottron's papules, and/or heliotrope rash, photosensitivity), and a chronic disease course with a low mortality. The frequency of skin ulcerations and lipodystrophy differs between the two cohorts

Objectives: To report the clinical and muscle histology associations of anti-TIF-1- $\gamma \mathrm{Ab}$ in a series of patients with JDM followed in the French referral center for rare pediatric systemic autoimmune diseases.

Methods: Retrospective study of patients with JDM (according to the EULAR/ACR criteria) associated with anti-TIF-1- $\gamma$ autoantibodies and included in our CEMARA database approved by the French National Committee on Informatics and Liberty.

Results: Thirteen patients were included (males: 5, females: 8; Caucasians: 6, Black Africans: 2, North Africans: 5). Age at diagnosis ranged from 1.5 to 11 years. Serum creatine kinase was elevated in 12 patients (range: 180-43 $000 \mathrm{IU} / \mathrm{L}$ ). Three different phenotypes were identified according to the severity and course. In group $1 \quad(n=4), 3$ patients had a moderate JDM: classical cutaneous manifestations and moderate muscle involvement; relapsing course, which remitted under methotrexate/corticosteroids; an additional 2-year-old girl had an amyopathic JDM. In group 2 $(n=7)$, patients had a severe JDM : ulcerating skin lesions, severe muscle involvement, dysphagia, gastrointestinal vasculitis and/or requirement to an intensive care unit, and required more than two lines of treatment. In this group, 2/7 patients died from refractory JDM, comparing to a mortality rate of $2 \%$ in the remaining JDM patients tested for MSA and negative for anti-TIF-1- $\gamma$. Only $2 / 5$ patients achieved a complete remission under treatment. Group 3 comprised two patients with severe muscle atrophy, calcinosis and lipodystrophy and a chronic course; one of them had a very-early-onset JDM at 1.5 year-old and the other an inherited neurological involvement, potentially suggestive of a genetic predisposing condition to JDM. None of the patients developed lung involvement or malignancy. Six patients underwent a muscle biopsy which was 
scored using the JDM severity score tool. The histo-pathologists' overall severity score (VAS) varied from to 1 to $9 / 10$; the highest scores were observed in the two patients who died.

Conclusion: In the present series of patients with JDM, TIF-1- $\gamma$ Ab-associated-clinical phenotypes and ethnicities are more heterogeneous than previously reported. TIF-1- $\gamma \mathrm{Ab}$ is associated with a high mortality rate in a subset of patients.

\section{REFERENCES}

[1] Rider LG, Nistala K. The juvenile idiopathic inflammatory myopathies: pathogenesis, clinical and autoantibody phenotypes, and outcomes. J Intern Med. 2016;280(1):24-38

Disclosure of Interests: Brigitte Bader-Meunier: None declared, cyril gitiaux: None declared, jean-luc charuel: None declared, Nicole Fabien: None declared, isabelle melki: None declared, Pierre Quartier Consultant for: AbbVie, Chugai-Roche, lilly, Novartis, Novimmune, Sanofi, and SOBI, Consultant for: AbbVie, Chugai-Roche, Lilly, Novartis, Novimmune, Sanofi, and SOBI, Speakers bureau: AbbVie, BMS, Chugai-Roche, Novartis, Pfizer, and SOBI, Speakers bureau: AbbVie, BMS, Chugai-Roche, Novartis, Pfizer, and SOBI, alexandre belot: None declared, Nathalie Streichenberger: None declared, christine bodemer: None declared, audrey aussy: None declared, olivier boyer: None declared

DOI: 10.1136/annrheumdis-2019-eular.3358

\section{AB0936 A NATIONAL SURVEY OF CLINICAL PRACTICE OF CORTICOSTEROID USE IN NEWLY DIAGNOSED OR FLARING CASES OF JUVENILE IDIOPATHIC ARTHRITIS ACROSS THE UK}

Eileen Baildam ${ }^{1}$, Ashley Jones ${ }^{2}$, Dannii Clayton ${ }^{2}$, Gloria Nkoma ${ }^{2}$, Matthew Peak ${ }^{1}$, Athimalaipet Ramanan ${ }^{3}$, Madeleine Rooney ${ }^{4}$, Helen Foster ${ }^{5}$, Simon Stones ${ }^{6}$, Flora Mcerlane ${ }^{7}$, Tracy Moitt ${ }^{2}$, Louise Roper ${ }^{8}$, Bridget Young ${ }^{8}$, Frances Sherratt ${ }^{8}$, Michael Beresford ${ }^{9}{ }^{1}$ Alder Hey Children's Hospital - Liverpool, Paediatric Rheumatology, Liverpool, United Kingdom; ${ }^{2}$ University of Liverpool, Clinical Trials Unit, Liverpool, United Kingdom; ${ }^{3}$ Bristol Royal Hospital for Children, Paediatric Rheumatology, Bristol, United Kingdom; ${ }^{4}$ Royal Belfast Hospital for Sick Children, Paediatric Rheumatology, Belfast, United Kingdom; ${ }^{5}$ University of Newcastle upon Tyne, Paediatric Rheumatology, Newcastle upon Tyne, United Kingdom; ${ }^{6}$ University of Leeds, School of Healthcare, Leeds, United Kingdom; ${ }^{7}$ Great North Children's Hospital, Paediatric Rheumatology, Newcastle upon Tyne, United Kingdom; ${ }^{8}$ University of Liverpool, Institute of Population Health Sciences, Liverpool, United Kingdom; ${ }^{9}$ University of Liverpool, Department of Translational Medicine, Liverpool, United Kingdom

Background: Corticosteroids (CS) are widely used for rapid-action or induction treatment in children and young people (CYP) with juvenile idiopathic arthritis (JIA). Given a lack of evidence base on CS induction regimen for CYP with JIA, and since criteria for choosing CS are based on healthcare professional (HCP) preference, further research is needed (1). Objectives: To establish the opinions of HCPs current practice regarding the clinical criteria for commencing CS treatment

Methods: A national electronic survey was undertaken among HCPs across the UK as part of the Steroid Induction Regimen for Juvenile Idiopathic Arthritis (SIRJIA) study.

Results: A total of $39(24 \%)$ responses were received from 162 HCPs. These included $22(56 \%)$ NHS consultants, five (13\%) grid trainees, eight (21\%) clinical nurse specialists and four other HCPs $(10 \%)$.

The most common treatments in CYPs with newly diagnosed JIA or a disease flare were intra-articular (IA) CS or a combination of DMARDs and IAS (except for systemic JIA and oligoarticular JIA). The majority of HCPs 17 would treat new and flaring CYP the same in terms of a CS remission induction regime, with $53 \%$ choosing a different regime or not answering.

The key criteria that HCPs used for commencing CS and choosing the route of administration were rapid induction of remission (31 (89\%)), high disease activity $(31(89 \%))$, severity of systemic JIA $(30(86 \%))$ and level of inflammation $(28(80 \%))$ Table 1 . The number one determinant of route of administration of CS was disease severity followed by disease subtype.

The majority of HCPs (52-72\% depending on role) would consider entering CYP with JIA into a trial randomising to the various modes of administration.
Table 1:

\begin{tabular}{lcc}
\hline Reasons of CS Choice & $\begin{array}{c}\text { Number } \\
\text { N=39 }\end{array}$ & Percentage\% \\
\hline High Disease Activity & 35 & 89.7 \\
Rapid induction of remission & 34 & 87.2 \\
Severity of Systemic JIA & 34 & 87.2 \\
Level of inflammation & 32 & 82.5 \\
Severe Uveitis & 30 & 76.9 \\
JIA subtype & 27 & 68.2 \\
Targeting Specific Joints & 26 & 66.7 \\
Level of Disability & 18 & 46.2 \\
Level of pain & 16 & 41.0 \\
Long-standing Disease & 11 & 28.1 \\
Patient reluctance to take DMARDS & 8 & 20.5 \\
\hline
\end{tabular}

Conclusion: The results from this national survey of clinical practice showed varying practices in the management of new CYP with JIA and those that are flaring. The majority of HCPs who completed this survey, indicated that they would be prepared to consider entering CYP into a trial that randomised to the four CS delivery methods.

\section{REFERENCES}

[1] Deepak, et al. 2017 Literature review on the use of corticosteroids in juvenile idiopathic arthritis (JIA) Rheumatology 56(Suppl7)

Acknowledgement: This study was funded by the NIHR Health Technology Assessment Programme (14/167/01). The funders did not have a role in the design, collection, analysis, or interpretation of the data Disclosure of Interests: Eileen Baildam Consultant for: EB has received one paid consultancy for Pfizer in the last 12 months, but this was not related to this study., Ashley Jones: None declared, Dannii Clayton: None declared, Gloria Nkoma: None declared, Matthew Peak: None declared, Athimalaipet Ramanan Consultant for: AbbVie, UCB, Sobi, Eli Lilly, Speakers bureau: Speaker fees/honoraria from Abbvie, SOBI, Eli Lilly and UCB, Speakers bureau: AbbVie, UCB, Sobi, Eli Lilly, Madeleine Rooney: None declared, Helen Foster: None declared, Simon Stones Consultant for: SS has provided consultancy services to Envision Pharma Group though this is not related to the contents of this abstract., Speakers bureau: SS has undertaken speaking engagements for Actelion, eyeforpharma, Four Health, Janssen and Roche, though these are not related to the contents of this abstract., Flora McErlane: None declared, Tracy Moitt: None declared, Louise Roper: None declared, Bridget Young: None declared, Frances Sherratt: None declared, Michael Beresford: None declared

DOI: 10.1136/annrheumdis-2019-eular.6751

\section{AB0937 IDENTIFYING THE PRIMARY OUTCOME MEASURE AND PROTOCOL COMPONENTS FOR A PROSPECTIVE FEASIBILITY STUDY OF CORTICOSTEROID REGIMENS FOR CHILDREN AND YOUNG PEOPLE WITH JUVENILE IDIOPATHIC ARTHRITIS USING CONSENSUS METHODS WITH YOUNG PEOPLE, FAMILIES AND PROFESSIONALS}

Simon Stones ${ }^{1}$, Heather Bagley ${ }^{2}$, Ashley Jones ${ }^{2}$, Flora Mcerlane ${ }^{3}$, Tracy Moitt ${ }^{2}$, Gloria Nkhoma ${ }^{2}$, Frances Sherratt ${ }^{4}$, Bridget Young ${ }^{4}$, Michael Beresford ${ }^{2,5}$, Eileen Baildam ${ }^{5}$, SIRJIA Trial Management Group. 'University of Leeds, School of Healthcare, Leeds, United Kingdom; ${ }^{2}$ University of Liverpool, Institute of Translational Medicine, Liverpool, United Kingdom; ${ }^{3}$ Newcastle Hospitals NHS Foundation Trust, Great North Children's Hospital, Newcastle upon Tyne, United Kingdom; ${ }^{4}$ University of Liverpool, Institute of Population Health Sciences, Liverpool, United Kingdom; ${ }^{5}$ Alder Hey Children's NHS Foundation Trust, Liverpool, United Kingdom

Background: Juvenile idiopathic arthritis (JIA) is an umbrella term for seven relapsing-remitting inflammatory conditions in children and young people (CYP). Early, intensive treatment can prevent long-term damage; however, established drugs exhibit a delayed response, prompting the need for rapid-onset treatment in the form of corticosteroids. Given a lack of consensus as to which corticosteroid induction regimen should be used for CYP with JIA, a feasibility trial of different regimens is needed. 